\title{
Comparison of maternal isocaloric high carbohydrate and high fat diets on osteogenic and adipogenic genes expression in adolescent mice offspring
}

Seyedeh Neda Mousavi ${ }^{1,4}$, Fariba Koohdani ${ }^{1 *}$, Farzad Shidfar ${ }^{2^{*}}$ and Mohamadreza Baghaban Eslaminejad ${ }^{3}$

\begin{abstract}
Background: Maternal high fat/high calorie diet leads to adiposity and bone fracture in offspring. However, the effects of macronutrient distribution in maternal isocaloric diet have not been studied. The present study was designed to test the hypothesis that maternal isocaloric pair-fed high-carbohydrate diet will increase osteoblastic and decrease osteoclastic and adipogenic gene expression compared with high-fat diet in adolescent mice offspring.

Methods: Virgin female C57BL/6 mice were impregnated and fed either the AIN 93G isocaloric pair-fed highcarbohydrate (LF-HCD) or a high fat (HF-LCD) diet from the time of vaginal plug confirmation until the offspring was weaned.

Results: After adjusting for the sex of offspring, osteoprotegrin (OPG) and Ctnnb1 (beta-catenin) genes expression were significantly reduced by $98 \%$ and $97 \%$ in the bone of offspring born from the HF-LCD compared with the LF-HCD-fed mothers ( $p<0.001$ and $p<0.001$, respectively). Peroxisome proliferator-activated receptor gamma-2 (PPAR $\gamma 2$ ) gene expression in the bone of offspring born from the HF-LCD was 7.1-folds higher than the LF-HCDfed mothers $(p=0.004)$. In the retroperitoneal fat mass of offspring born from HF-LCD, AdipoQ and LPL genes expression were respectively up-regulated 15.8 and 4.2-folds compared with the LF-HCD-fed mothers $(p<0.001$ and $p=0.03$, respectively).
\end{abstract}

Conclusions: Maternal isocaloric pair-fed high-carbohydrate diet enhances osteoblastogenesis and inhibits adipogenesis compared with high-fat diet in adolescent mice offspring.

Keywords: Osteoblastogenesis, Adipogenesis, Macronutrients, Isocaloric diet, Mice

\section{Background}

Obesity and osteoporosis have increased globally [1, 2]. Adipose tissue is an endocrine gland that affects bone metabolism [3]. In recent years, the importance of the association between fat and bone in the pathophysiology of bone loss has been highlighted [4]. Osteoblasts and adipocytes are derived from a common multipotential

\footnotetext{
* Correspondence: fkoohdan@tums.ac.ir; shidfar.f@iums.ac.ir

1 Department of Cellular and Molecular Nutrition, School of Nutritional Sciences and Dietetics, Tehran University of Medical Sciences, Tehran, Iran

${ }^{2}$ Department of Nutrition, School of Health, Iran University of Medical Sciences, Tehran, Iran

Full list of author information is available at the end of the article
}

mesenchymal stem cell (MSC) progenitor [5]. Bone is constantly remodeled throughout the process of bone formation by osteoblasts and bone resorption by osteoclasts [6]. Developmentally, osteoclasts are derived from hematopoietic stem cell precursors of the monocyte/ macrophage lineage in the blood and the bone marrow, while osteoblasts originate from bone marrow mesenchymal stem cells [7]. Considerable evidence exists to support that a shift in MSC differentiation to favor the adipocyte over the osteoblast lineage can directly contribute to imbalances in bone formation/resorption and ultimately leads to bone loss. This shift of MSC differentiation to the adipocyte lineage may contribute to the 
progressive increase in adipocyte formation and decrease in osteoblast number that coincides with age-related bone loss [8]. Adipocytes may further influence on bone remodeling through the secretion of adipokines with paracrine actions that may influence the development and function of stem cell precursors as well as mature cell types such as osteoblasts and osteoclasts [9]. Thus, given the close association between adipocyte and osteoblast formation, the potential exists to prevent or treat bone loss by inhibiting bone marrow adipogenesis.

Unbalanced feeding is one of the most determinant factors that can induce metabolic disorders [10]. In this context, a number of experimental studies have demonstrated that not only the energy intake, but also the macronutrient distribution of the diet may play an important role in obesity, diabetes, hypertension and other metabolic disorders $[11,12]$. Macronutrient distribution in maternal diet during pregnancy may affect body composition of the offspring later in life, but evidence is still scarce [13]. Evidence from experimental models clearly demonstrate that maternal obesity, independent of birth weight, leads to developmental programming of adiposity gain in the offspring $[14,15]$. Unfortunately there are currently no explanations, either clinically or experimentally, to assess the macronutrient composition in maternal isocaloric diet during gestation and lactation on osteoblastic, osteoclastic and adipogenic genes expression in adolescent mice offspring. Therefore, this study was designed to investigate the different effects of maternal isocaloric low fat-high carbohydrate diet (LF-HCD) and high fat-low carbohydrate diet (HF-LCD) on osteoblastic, osteoclastic and adipogenic genes expression in adolescent mice offspring. We hypothesized that isocaloric pair-fed HF-LCD induces osteoclastogenesis and adipogenesis compared with LF$\mathrm{HCD}$ in adolescent offspring. To achieve this, virgin female C57BL/6 mice were used as a model to study the effects of two isocaloric diets containing different amounts of fat and carbohydrate during gestation and lactation on bone osteoblastic, osteoclastic and adipogenic gene expression levels in adolescent male or female offspring.

\section{Methods}

The experimental protocol was approved by the Animal Research Committee of Iran University of Medical Sciences (protocol number: 24208). This research conforms to the Institutional and National Guide for the Care and Use of Laboratory Animals. Eight-week-old inbred female C57BL/6 mice $(21 \pm 1.5 \mathrm{~g})$ were obtained from the Razi Vaccine and Serum Research Institute, Tehran, Iran. Each mouse was individually housed at $21-23{ }^{\circ} \mathrm{C}$ with controlled humidity $(50 \pm 5 \%)$ under a $12 \mathrm{~h}$ artificial light cycle (7 am to $7 \mathrm{pm}$ ). The AIN $93 \mathrm{M}$ diet composition (per $1 \mathrm{~kg}$ ) was $140 \mathrm{~g}$ of protein as Casein lactate (Iranian Caseinate Industry, Iran) and $1.8 \mathrm{~g}$ as L-cystein (W326305, Sigma Aldrich, Germany), $40 \mathrm{~g}$ of lipid as soybean oil (Kamzit Company, Iran), $630 \mathrm{~g}$ of carbohydrate as corn starch (corn dextrin from corn refinery, Iran), $100 \mathrm{~g}$ as sugar (local confectionery, Iran), $35 \mathrm{~g}$ of AIN 93 M mineral mix (296040002, MP Biomedicals, USA), $10 \mathrm{~g}$ of AIN 93 vitamin mix (296040201, MP Biomedicals, USA), $2.5 \mathrm{~g}$ of choline bitartrate (C1629, Sigma Aldrich, Germany), $0.008 \mathrm{~g}$ of tert-butyl hydroquinone (112941, Sigma Aldrich, Germany) and $50 \mathrm{~g}$ of fiber (wheat bran, Iran).

Experimental timeline was shown in Fig. 1. Each female mouse was mated with one male per cage overnight. After vaginal plug confirmation, mothers were randomly assigned to dietary groups $(N=10$ in each group), as shown in Table 1. The LF-HCD is considered as the control diet group.

During the study, all animals were isocalorically fed with the same $\mathrm{kcal} /$ [g body weight] of the group that

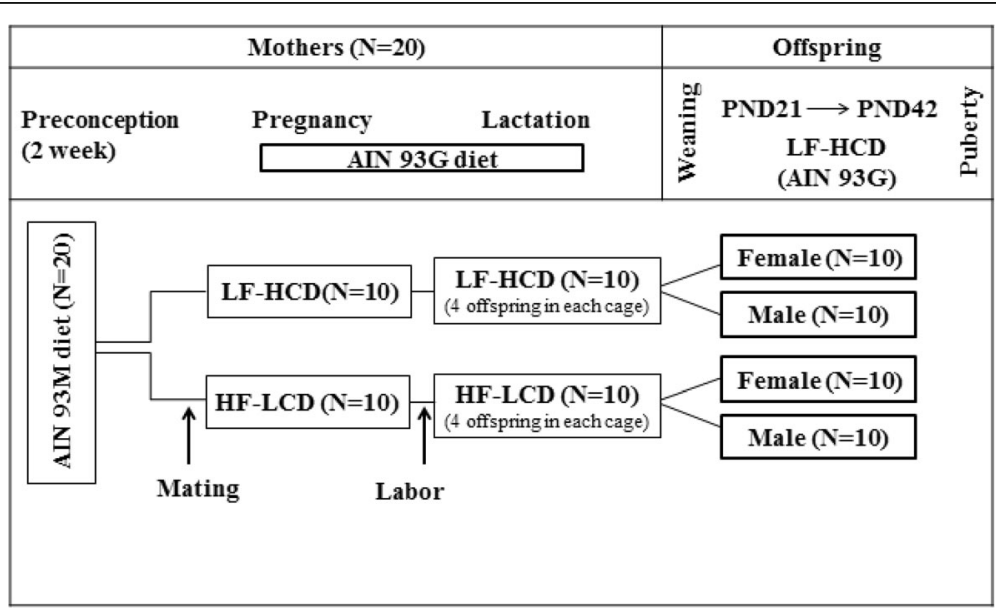

Fig. 1 Experimental timeline. LF-HCD: low fat-high carbohydrate diet; HF-LCD: high fat-low carbohydrate diet 
Table 1 Composition of the experimental diets per $1 \mathrm{~kg}$ during the study (AIN 93G diet)

\begin{tabular}{|c|c|c|}
\hline Diets & LF-HCD & HF-LCD \\
\hline \multicolumn{3}{|l|}{ Nutrients (g/kg) } \\
\hline Casein & 200 & 200 \\
\hline Cornstarch & 530 & 247 \\
\hline Sucrose & 100 & 100 \\
\hline Soy oil & 70 & 198 \\
\hline Fiber & 50 & 204.5 \\
\hline Mineral mix & 35 & 35 \\
\hline Vitamin mix & 10 & 10 \\
\hline$L-c y s^{a}$ & 3 & 3 \\
\hline Choline bitartrate & 2.5 & 2.5 \\
\hline tert-butyl hydroquinone & 0.008 & 0.008 \\
\hline Energy (kcal/gr) & 3.97 & 3.97 \\
\hline
\end{tabular}

LF-HCD low fat-high carbohydrate diet, HF-LCD high fat-low carbohydrate diet a'- cysteine

ate less (pair-fed model) [16]. Offspring were reduced to 4 pups in each cage, nursed by birth mothers and weaned on day 21, when they were separated according to sex with a maximum of four mice per cage. Pups received ad libitum control diet post-weaning. One male and one female offspring were randomly selected from each cage for final gene expression analysis at 6 weeks of age.

\section{Weight and biochemical measures}

Animals were weighed on a calibrated balance scale (Marte Scale (EK-3000i, USA)) at birth and adolescence. All pups were killed at 6 weeks of age by administration of $40 \mathrm{mg} / \mathrm{kg}$ ketamine and $8 \mathrm{mg} / \mathrm{kg}$ xylazine, following the overnight fasting conditions. Blood samples were collected at 08.00 a.m., after overnight fasting from the heart. All the biochemical parameters were measured by autoanalyzer. Serum OPG (R\&D system; Cat $\neq$ DY459), RANK-L (R\&D system; Cat $\neq$ MTR00), Lep (R\&D system; Cat $\neq$ MOB00) and AdipoQ (EMD Millipore; Cat $\neq$ EZMADP-60 K) were measured by ELISA method, according to the manufacturer's instructions.

\section{Collection and preparation of specimens}

The right femur and retroperitoneal fat were dissected out, stripped of soft tissue using a scalpel, immediately frozen and then were powdered.

\section{RNA isolation and real-time polymerase chain reaction (RT-PCR)}

Total RNA from the powdered samples were extracted using QIAzol Lysis Reagent (QIAGEN Inc., Valencia, CA 91355, USA) according to the manufacturer's instructions, followed by DNase digestion and column cleanup using RNeasy mini columns (Qiagen, Valencia, CA, USA) [17]. RNA quantification was assessed at $260 \mathrm{~nm}$ with a ND-1000 spectrophotometer (NanoDrop, Wilmington, DE, USA), and its quality (integrity) was checked by agarose gel electrophoresis. One microgram of total RNA was retro-transcribed into cDNA using PrimeScript cDNA synthesis reagents from Takara (Takara Bio, Inc., Japan) in $20 \mu \mathrm{l}$ volume. The RT-PCR was performed in an ABI StepOne sequence detection system (Applied Biosystems, California, USA) with $1 \mu \mathrm{l}$ of cDNA and 10 pmol of each primer corresponding to the tested genes, using the SYBR Green I Master Mix (Roche). The expression assay was run in duplicate. Primer pairs used were shown in Table 2.

All primers for RT-PCR analysis were designed using Primer Express software 2.0.0 (Applied Biosystems). The relative quantity of mRNA was calculated for each sample using the copy threshold $(\mathrm{Ct})$ value and normalized against glyceraldehyde 3-phosphate dehydrogenase (GAPDH, housekeeping gene) mRNA. The stability of

Table 2 Real-time-PCR primer sequences

\begin{tabular}{|c|c|c|}
\hline Gene & Forward primer & Reverse primer \\
\hline$\overline{O P G}$ & 5'-GGGCGTTACCTGGAGATCG-3' & 3'-CGTTGTCATGTGTTGCATTTCC-5' \\
\hline RANKL & 5'-CAGCATCGCTCTGTTCCTGTA-3' & 3'-CTGCGTITCATGGAGTCTCA-5' \\
\hline Ctnnb1 & 5'-CCTCCCAAGTCCTTTATGAATGG-3' & 3'-CCGTCAATATCAGCTACTTGCTCTT-5' \\
\hline PPARY2 & 5'-GCCCTTTGGTGACTITATGGA-3' & 3'-GCAGCAGGTTGTCTTGGATG-5' \\
\hline$L P L$ & 5'- GGGAGTTTGGCTCCAGAGTTT-3' & 3'- CGTGTGTGAAATGTCATTGATCC-5' \\
\hline Lep & 5'- GTGGCTTTGGTCCTATCTGTC-3' & 3'- CGTGTGTGAAATGTCATTGATCC-5' \\
\hline AdipoQ & 5'- TGTTCCTCTTAATCCTGCCCA-3' & 3'- CCAACCTGCACAAGTTCCCTT-5 \\
\hline FASN & 5'- AGAGATCCCGAGACGCTTCT-3' & 3'-GCTTGGTCCTTTGAAGTCGAAGA-5' \\
\hline Runx2 & 5'- CAGCATCCTATCAGTTCCCAA-3' & 5'- CAGCGTCAACACCATCATT-3' \\
\hline GAPDH & 5'- GACTTCAACAGCAACTCCCAC-3' & 3'- TCCACCACCCTGTTGCTGTA-5' \\
\hline
\end{tabular}

OPG osteoprotegrin, RANK-L Receptor activator of nuclear factor kappa-B ligand, Ctnnb1 beta-catenin, PPAR $\gamma 2$ Peroxisome proliferator-activated receptor gamma2, LPL lipoprotein lipase, Lep leptin, AdipoQ adiponectin, FASN fatty acid synthase, Runx2 Runt-related gene 2, GAPDH glyceraldehyde 3-phosphate dehydrogenase 
the housekeeping gene was considered a $\mathrm{Ct}$ standard deviation $<1$ and was therefore considered an appropriate control [18]. The amplification profile included one cycle at $95{ }^{\circ} \mathrm{C}$ for $10 \mathrm{~min}$ and 40 two-step cycles: $95{ }^{\circ} \mathrm{C}$ for $15 \mathrm{~s}$ and $60{ }^{\circ} \mathrm{C}$ for $60 \mathrm{~s}$. The results were generated

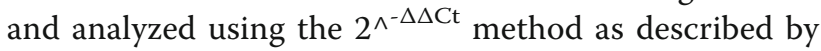
Livak and Schmittgen [19].

\section{Statistical analyses}

In the designing of the study, to detect a difference in total body fat mass between two groups, we used a $90 \%$ confidence interval with a two-sided test with $\alpha=0.05$ (type I error). On the basis of SDs reported in a similar study [20], the number of subjects needed to detect this difference was 16 per group. Data were tested for normal distribution using the Kolmogorov-Smirnov test. Independent sample $t$-test was used to compare the variables between two groups. Data did not have normal distributions for gene expression even after all of the transformation methods. The differences between two variables were measured by the Mann-Whitney Wilcoxon test, controlling for the sex of offspring. To assess the interactions between sex and diets on the target gene expression, a logistic regression model was used in which any gene was coded as a nominal variable (gene expression above the 50th percentile or below the 50th percentile) [21]. All data are expressed as the means \pm SE. The level of significance was set at $P<0.05$. Statistical analyses were performed with the IBM SPSS Statistics software (version 18; IBM Corp).

\section{Results}

The average of food intake was not significantly different between the LF-HCD versus HF-LCD group at the end of weeks $1(2.4 \pm 0.17 \mathrm{~g} /$ day vs. $2.44 \pm 0.2 \mathrm{~g} /$ day $)$, week 2 $(2.99 \pm 0.18 \mathrm{~g} /$ day vs. $2.92 \pm 0.12 \mathrm{~g} /$ day $)$ or week $3(5.94$ $\pm 0.27 \mathrm{~g} /$ day vs. $5.91 \pm 0.2 \mathrm{~g} /$ day), post-weaning. In the female offspring, adolescence weight was significantly higher in the LF-HCD than HF-LCD group $(p<0.001)$. Serum HDL.C was significantly higher in the HF-LCD than LF-HCD group $(p=0.006)$. In male offspring, there were significant differences in birth and adolescence weight ( $p=0.008$ and $p=0.01$, respectively). Also, serum glucose was significantly higher in male than females in the LF-HCD group $(p=0.005)$. In the HF-LCD group, there were significant differences in adolescence weight, TC, LDL.C and TG between males and females ( $p<0.001, p=0.008, p=0.01$ and $p=0.002$, respectively) (Table 3).

As shown in Figs. 2 and 3, Runx2 and Ctnnb1 gene expression were significantly higher in female offspring born from the LF-HCD compared with the HF-LCD-fed mothers $(p<0.001$ and $p=0.01$, respectively). In male offspring, mRNA expression of Runx2, OPG, OPG/
Table 3 Weight and biochemical measures in the male and female offspring of dietary groups ${ }^{a}$

\begin{tabular}{|c|c|c|c|c|}
\hline Variables & Sex & LF-HCD & HF-LCD & $p$ value \\
\hline \multirow[t]{2}{*}{ Birth weight (g) } & Female & $1.29 \pm 0.31^{b}$ & $1.3 \pm 0.13$ & 0.9 \\
\hline & Male & $1.7 \pm 0.23$ & $1.3 \pm 0.12$ & $<0.001^{*}$ \\
\hline \multirow[t]{2}{*}{ Adolescent weight (g) } & Female & $19.7 \pm 1.06^{b}$ & $17.4 \pm 1.4^{c}$ & $0.002^{*}$ \\
\hline & Male & $21.3 \pm 1.3$ & $21.8 \pm 0.79$ & 0.3 \\
\hline \multirow[t]{2}{*}{$\mathrm{TC}(\mathrm{mg} / \mathrm{dl})$} & Female & $177.1 \pm 61.4$ & $146.1 \pm 16.5^{c}$ & 0.2 \\
\hline & Male & $207.5 \pm 81.3$ & $205.4 \pm 51.9$ & 0.9 \\
\hline \multirow[t]{2}{*}{ LDL.C (mg/dl) } & Female & $93.3 \pm 51.5$ & $54.7 \pm 19.9^{c}$ & 0.08 \\
\hline & Male & $111.2 \pm 65.6$ & $89.9 \pm 37.05$ & 0.4 \\
\hline \multirow[t]{2}{*}{ HDL.C (mg/dl) } & Female & $31.9 \pm 10.4$ & $48 \pm 9.6$ & $0.006^{*}$ \\
\hline & Male & $42.8 \pm 11.6$ & $49.2 \pm 18.9$ & 0.4 \\
\hline \multirow[t]{2}{*}{ Triglyceride (mg/dl) } & Female & $179.6 \pm 97.4$ & $156.7 \pm 24.9^{c}$ & 0.2 \\
\hline & Male & $266.8 \pm 132.8$ & $273.1 \pm 69.7$ & 0.9 \\
\hline \multirow[t]{2}{*}{ Glucose (mg/dl) } & Female & $136 \pm 23.6^{b}$ & $118.7 \pm 33.2$ & 0.2 \\
\hline & Male & $183.8 \pm 32.8$ & $153 \pm 67.7$ & 0.3 \\
\hline
\end{tabular}

$p$ values are calculated with the independent sample $t$-test; TC total cholesterol

${ }^{a}$ All data reported as mean $\pm S D$; ${ }^{b}$ significant difference between male and female offspring in the LF-HCD group $(p \leq 0.01)$; ${ }^{c}$ significant difference between male and female offspring in the HF-LCD group $(p \leq 0.01)$ ${ }^{*}$ significant differences between LF-HCD and HF-LCD group

RANK-L ratio and Ctnnb1 were significantly higher in the offspring born from LF-HCD than HF-LCD-fed mothers $(p<0.001, p<0.001, p<0.001$ and $p=0.03$, respectively). In contrast, PPAR 2 gene expression was significantly higher in female and male offspring of the HF-LCD than LF-HCD group ( $p=0.04$ and $p<0.001$, respectively). LPL, Lep, AdipoQ and FASN genes expression were significantly higher in female, as well as in male offspring born from HF-LCD compared with the LF-HCD-fed mothers $(p<0.001, p<0.001, p<0.001$ and $p<0.001$, respectively). (Figs. 4 and 5)

According to the results of the logistic regression model, the diets showed significant effects on OPG, Ctnnb1, PPAR 2 genes expression in the bone $(p<$ $0.001, p<0.001$ and $p=0.004$, respectively), as well as LPL and AdipoQ genes expression in the retroperitoneal fat ( $p=0.03$ and $p<0.001$, respectively), after being adjusted for the sex of offspring. OPG and Ctnnb1 genes expression were significantly reduced by $98 \%$ and $97 \%$ in the bone of offspring born from the HFLCD compared with the LF-HCD-fed mothers. PPAR 2 gene expression in the bone of offspring born from the HF-LCD was 7.1-folds higher than the LFHCD-fed mothers. In the retroperitoneal fat mass of offspring born from HF-LCD, AdipoQ and LPL genes expression were respectively up-regulated 15.8 and 4.2folds compared with the LF-HCD-fed mothers. Adjusting for the diets, the sex of offspring had significant effect on OPG/RANK-L expression ratio $(p=0.02)$. OPG/ 


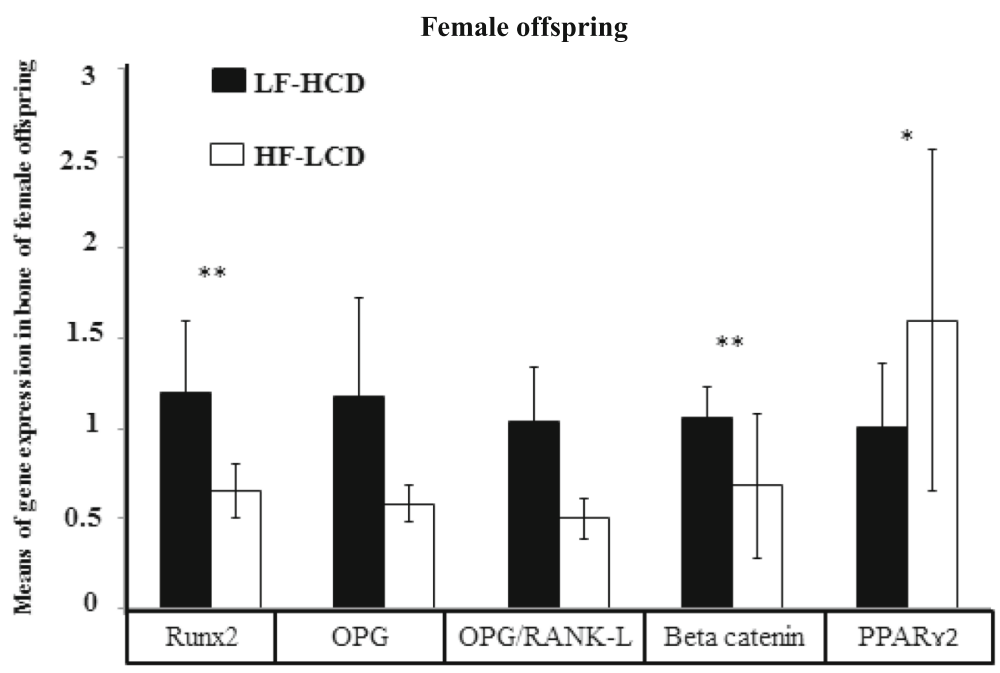

Fig. 2 Means of bone genes expression in adolescent female mice offspring. Runx2 and Ctnnb1 gene expression were significantly higher in the LF-HCD than the HF-LCD group. In contrast, PPARx2 gene expression was significantly higher in the HF-LCD group $\left({ }^{*} p<0.05 ;{ }^{* *} p<0.001\right)$

RANK-L expression ratio was significantly up-regulated by $80 \%$ in the bone of female compared to male offspring (Table 4).

In female offspring, serum level of RANK-L was significantly higher in the LF-HCD than HF-LCD group $(p<0.001)$. But, serum levels of Lep and AdipoQ were significantly lower in the LF-HCD than HF-LCD group ( $p<0.001$ and $p<0.001$, respectively). In male offspring, Serum level of OPG was significantly higher in the LFHCD than HF-LCD group $(p<0.001)$, but Lep protein level was significantly lower in the LF-HCD than HFLCD group $(p<0.001)$ (Fig. 6).

Serum OPG/RANK-L ratio was significantly higher in the male and female offspring of LF-HCD than HF-LCD group ( $p=0.01$ and $p=0.02$, respectively).

According to the results of the logistic regression model, the diets showed significant effects on serum OPG/RANK-L ratio, adjusted for the sex of offspring $(p=0.04)$. Serum OPG/RANK-L ratio was 5.5 -folds higher in the offspring born from LF-HCD than HF-

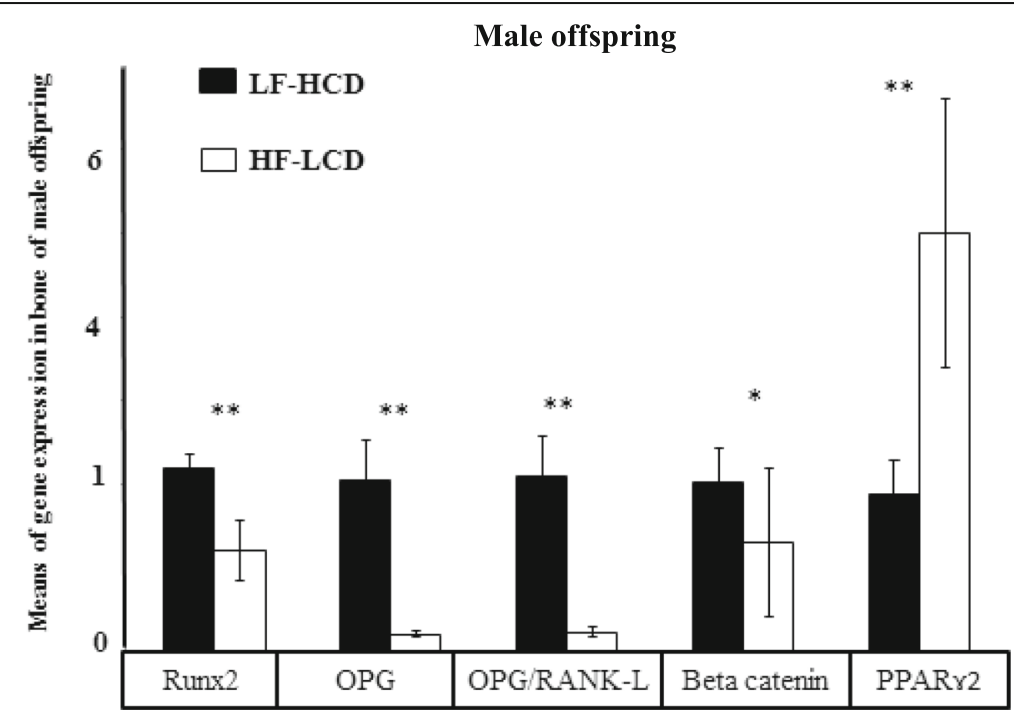

Fig. 3 Means of bone genes expression in adolescent male mice offspring. Runx2, OPG, OPG/RANK-L and Ctnnb1 genes expression were significantly higher in the LF-HCD than the HF-LCD group. In contrast, PPARr2 gene expression was significantly higher in the HF-LCD group $\left({ }^{*} p<0.05 ;{ }^{* *} p<0.001\right)$ 


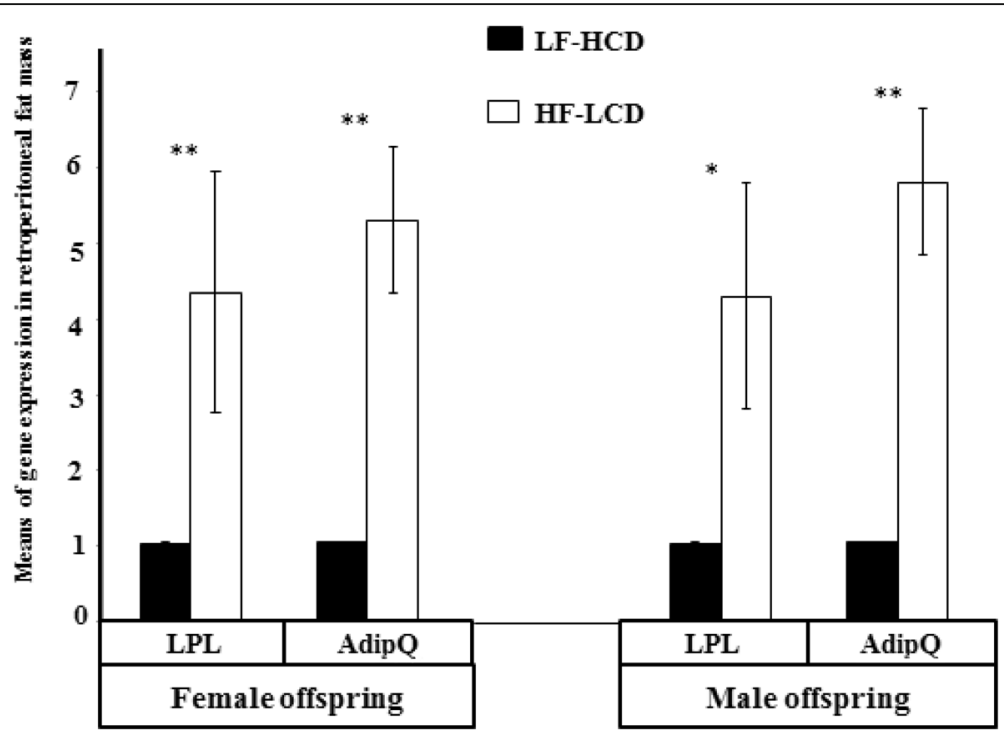

Fig. 4 Means of LPL and AdipoQ genes expression in adolescent female and male mice offspring. LPL and AdipoQ genes expression were significantly higher in the HF-LCD than the HF-LCD group $\left({ }^{*} p<0.05 ;{ }^{* *} p<0.001\right)$

LCD-fed mothers. After adjusting for the diets, serum OPG/RANK-L ratio was significantly higher in the females than males $(p<0.001)$. Serum OPG/RANK-L ratio increased by $96 \%$ in the females than males, adjusted for the diets.

\section{Discussion}

To our knowledge, these are the first results that demonstrate the effects of maternal isocaloric pair-fed highcarbohydrate (LF-HCD) versus high-fat diet (HF-LCD) during gestation and lactation on gene expression and serum levels of formation and resorption markers in bone, as well as adipogenic and lipogenic markers in retroperitoneal fat mass of mice offspring at adolescence. The results of the present study showed that maternal LF-HCD during gestation and lactation lead to up-regulation of Runx2 and Ctnnb1, as well as Runx2, OPG, OPG/RANK-L ratio and Ctnnb1 mRNA expression in bone of female and male offspring, respectively. Also, serum levels of OPG/RNK-L ratio which is the marker of osteogenesis [22] were increased in the LF-HCD-fed group, compared with the HF-LCD. PPAR 2 mRNA expression, as well as other adipogenic genes measured in the current study and serum levels of proteins were increased in the offspring of HF-LCDfed mothers. Our results showed that mRNA

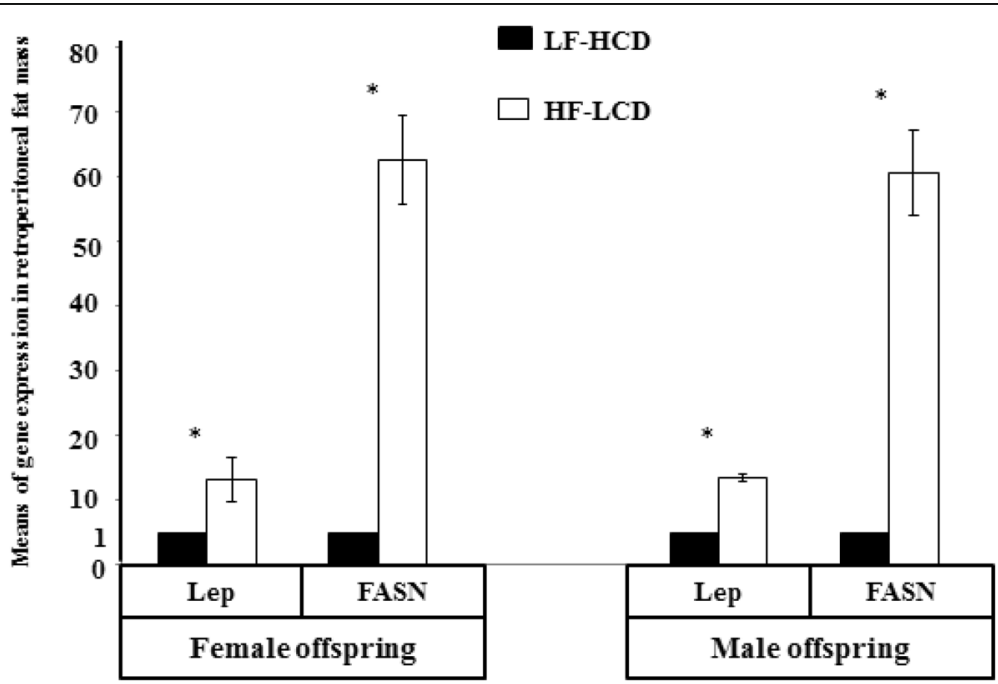

Fig. 5 Means of Lep and FASN genes expression in adolescent female and male mice offspring. Lep and FASN genes expression were significantly higher in the HF-LCD than the LF-HCD group $\left({ }^{*} p<0.05 ;{ }^{* *} p<0.001\right)$ 
Table 4 Status of the target gene expression, diets and sex of offspring

\begin{tabular}{|c|c|c|c|c|c|c|}
\hline \multirow{2}{*}{$\frac{\text { Gene }}{\text { Runx2 }}$} & \multicolumn{2}{|c|}{ Variable's level } & \multirow{2}{*}{$\begin{array}{l}\mathrm{B}^{\mathrm{a}} \\
-\end{array}$} & \multirow{2}{*}{$\frac{S E}{-}$} & \multirow{2}{*}{$\frac{p \text { value }}{0.9}$} & \multirow{2}{*}{$\frac{\mathrm{OR}(95 \% \mathrm{Cl})}{1}$} \\
\hline & Diets & LF-HCD & & & & \\
\hline & & HF-LCD & -40.6 & 1.3 & & $0.001(0.001,0.001)$ \\
\hline & Sex & Female & - & - & 0.9 & 1 \\
\hline & & Male & -18.4 & 9.1 & & $0.001(0.001,0.001)$ \\
\hline \multirow[t]{4}{*}{ OPG } & Diets & LF-HCD & - & - & $<0.001^{*}$ & 1 \\
\hline & & HF-LCD & -3.7 & 0.95 & & $0.02(0.004,0.16)$ \\
\hline & Sex & Female & - & - & 0.19 & 1 \\
\hline & & Male & 1.2 & 0.89 & & $3.2(0.55,18.3)$ \\
\hline \multirow[t]{4}{*}{ OPG/RANK-L } & Diets & LF-HCD & - & - & 0.12 & 1 \\
\hline & & HF-LCD & -1.01 & 0.65 & & $0.36(0.1,1.31)$ \\
\hline & Sex & Female & - & - & $0.02^{*}$ & 1 \\
\hline & & Male & -1.6 & 0.7 & & $0.2(0.05,0.78)$ \\
\hline \multirow[t]{4}{*}{ Ctnnb1 } & Diets & LF-HCD & - & - & $<0.001^{*}$ & 1 \\
\hline & & HF-LCD & -3.5 & 0.88 & & $0.03(0.005,0.17)$ \\
\hline & Sex & Female & - & - & 0.6 & 1 \\
\hline & & Male & 0.44 & 0.82 & & $1.5(0.31,7.73)$ \\
\hline \multirow[t]{4}{*}{ PPAR $r 2$} & Diets & LF-HCD & - & - & $0.004^{*}$ & 1 \\
\hline & & HF-LCD & 1.95 & 0.68 & & $7.1(1.83,27.1)$ \\
\hline & Sex & Female & - & - & 0.13 & 1 \\
\hline & & Male & 1.04 & 0.69 & & $2.8(0.73,11.1)$ \\
\hline \multirow[t]{4}{*}{ FASN } & Diets & LF-HCD & - & - & 0.99 & 1 \\
\hline & & HF-LCD & 21.9 & 817 & & $0.001(0.001,0.001)$ \\
\hline & Sex & Female & - & - & 0.56 & 1 \\
\hline & & Male & 0.51 & 0.87 & & $1.66(0.3,9.3)$ \\
\hline \multirow[t]{4}{*}{ AdipoQ } & Diets & LF-HCD & - & - & $<0.001^{*}$ & 1 \\
\hline & & HF-LCD & 2.8 & 0.74 & & $15.8(3.7,67.4)$ \\
\hline & Sex & Female & - & - & 0.4 & 1 \\
\hline & & Male & -0.62 & 0.74 & & $0.53(0.125,2.3)$ \\
\hline \multirow[t]{4}{*}{ Lep } & Diets & LF-HCD & - & - & 0.99 & 1 \\
\hline & & HF-LCD & 40.1 & 102 & & $0.001(0.001,0.001)$ \\
\hline & Sex & Female & - & - & 0.99 & 1 \\
\hline & & Male & -19.7 & 72 & & $0.001(0.001,0.001)$ \\
\hline \multirow[t]{4}{*}{ LPL } & Diets & LF-HCD & - & - & $0.03^{*}$ & 1 \\
\hline & & HF-LCD & 1.44 & 0.68 & & $4.2(1.1,16.2)$ \\
\hline & Sex & Female & - & - & 0.87 & 1 \\
\hline & & Male & 0.11 & 0.66 & & $1.1(0.3,4.1)$ \\
\hline
\end{tabular}

$p$ values are calculated with the logistic regression model LF-HCD low fat-high carbohydrate diet, HF-LCD high fat-low carbohydrate diet, $O R$ odds ratio, $C l$ confidence interval, $O P G$ osteoprotegerin, RANK- $L$ nuclear factor (NF-kB) ligand, PPRR 22 Peroxisome proliferator-activated receptor gamma 2, FASN fatty acid synthase, AdipoQ adiponectin, Lep leptin, LPL lipoprotein lipase

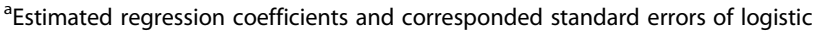
regression model

*significant differences adjusted for maternal diet and/or sex of offspring expression of OPG and Ctnnb1 were reduced in the bone of offspring born from the HF-LCD compared with the LF-HCD-fed mothers, adjusted for the sex. In contrast, PPARr2 gene expression was increased. Adjusted for the diets, OPG/RANK-L expression ratio was higher in the female than male offspring. Notably also, adjusted for the sex of offspring, serum levels of OPG/ RANK-L ratio was significantly increased in the LFHCD vs. HF-LCD group which result in osteogenesis. OPG and RANK-L are respectively determinants of osteoblastogenesis and osteoclastogenesis, in which OPG can block the effects of RANK-L [23]. Increase in OPG/ RANK-L ratio is a determinant of osteoblastogenesis. Runx2 is a transcriptional factor which modulates many aspects of skeletal development [24]. In another hand, Ctnnb1 signaling is necessary for osteoblast and osteocyte expression of OPG and induce osteogenesis [25]. Ctnnb1 inhibits adipogenesis in mesenchymal stem cell lineage, apoptosis in osteoblasts and differentiation of monocytes to osteoclasts [26]. Similar to OPG/RANK-L ratio, we observed that maternal LF-HCD during gestation and lactation lead to Runx2 and Ctnnb1 upregulation compared with the HF-LCD. At protein level, serum OPG/RANK-L ratio was significantly higher in the offspring born from LF-HCD-fed mothers than HF-LCD group. Adjusted for the diets, OPG/ RANK-L expression ratio was higher in female than male offspring, but sex had no significant effect on the expression of other target genes. Previous studies mentioned that $17 \beta$-estradiol enhances OPG gene expression and protein secretion through a transcriptional mechanism in MSCs [27, 28]. PPAR 2 is the dominant regulator of adipogenesis which its activation favors the differentiation of MSCs into adipocytes rather than osteoblasts [29]. Our results showed that maternal HF-LCD during gestation and lactation lead to PPAR 2 up-regulation in the bone of offspring, compared with LF-HCD. Accumulating data suggest that excessive fat mass is detrimental to bone [30, 31]. Although, there is a substantial body of evidence that maternal high calorie/high fat diet affect bone metabolism, growth, remodeling, and turnover [32], currently there is no evidence available on the effects of maternal isocaloric diet containing different amounts of fat and carbohydrate on bone.

LPL and FASN are adipogenic and lipogenic markers which are quantitatively important for synthesis and deposition of fatty acids in adipose tissues. Studies have shown that LPL and FASN over-expression result in higher fat mass and more insulin resistance [33, 34]. Leptin and adiponectin are the fat-derived hormones which effect centrally or peripherally on bone, that antagonize each other. Studies have shown that hypothalamic pathways of these adipokins are stronger than 


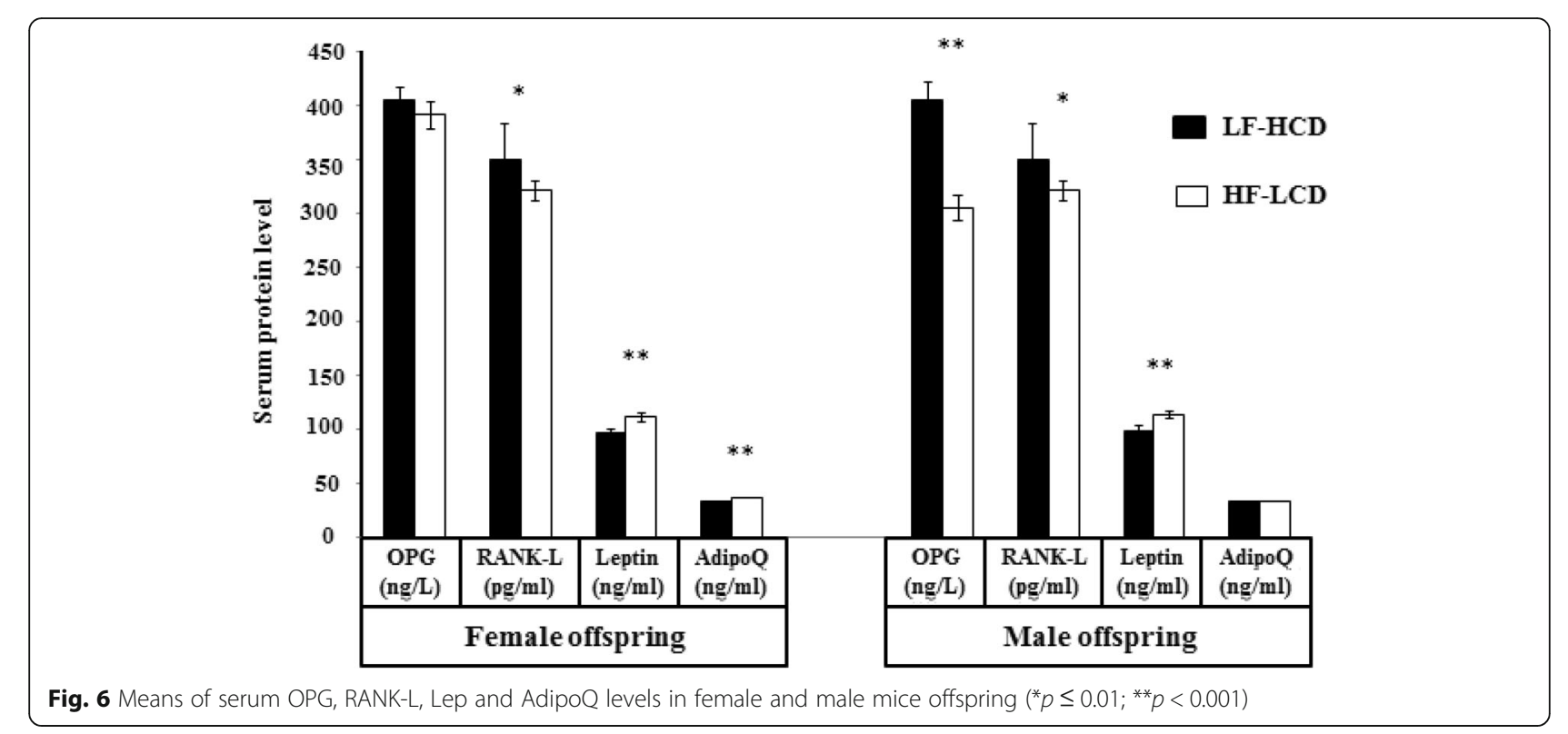

peripheral effects [35]. Leptin suppresses osteoblast activity and increases osteoclast numbers via RANK-L and b2-adrenergic receptor (Adrb2) [36, 37]. Adiponectin acts directly in osteoblasts to prevent their proliferation and increase their apoptosis in a PI3-kinase-FoxO1dependent manner in young ages. RANK-L expression was increased more than 10 -folds after stimulation by even low amounts of adiponectin or its globular domain. These results identifying the osteoblast and RANK-L as adiponectin target cell and gene implied that this hormone should inhibit bone mass accrual by favoring bone resorption. Over the time, by aging, this local effect obscured through sympathetic nervous system inhibition [38]. Results of our study are in agreement with these concepts. Adiponectin, Lep, FASN and LPL genes expression were increased in offspring born from the HF-LCD compared with the LF-HCD-fed mothers. In opposite OPG and Ctnnb1 as the markers of osteogenesis were decreased. Then, adiponectin signals back into osteoblasts and hamper their proliferation and favor their apoptosis in young age (6 weeks old). At protein level, serum leptin and adiponectin were significantly higher in the female offspring of HF-LCD-fed mothers than LF-HCD. In male offspring, serum leptin level was significantly higher in the HF-LCD-fed group. These results suggest that alterations in gene expression occur at protein level which is functional.

In summary, in the same calorie contents, LF-HCD is preferred to HF-LCD for maternal consumption during gestation and lactation due to increases in osteogenesis and decreases in adipogenesis in the next generation. Then, not only calorie content but also, macronutrient distribution in maternal diet during gestation and lactation is important. Therefore, our hypothesis mentioned at the beginning of the essay will be accepted.

There are some limitations to our data, most importantly, the fact that the background features of maternal mice fed with LF-HCD vs. HF-LCD should be provided. Skeletal and adipose tissue gene expression were assessed only at adolescence, even though periodic measures from birth until aging, is recommended. This study does not directly measure whether maternal LF-HCD and HF-LCD during gestation and lactation induce epigenetic changes on bone and fat mass of offspring or not. In future studies, direct assessment of epigenetic changes such as histone modification or DNA methylation of the mentioned target genes will allow us to test the hypothesis that perinatal programming of the skeleton occurs through epigenetic mechanisms. Additionally, genes expression was normalized against GAPDH as the housekeeping gene. Other housekeeping genes other than GAPDH are suggested for future studies. A free-fed group along with the pair-fed groups is also suggested for the future. This is an animal model; therefore, the effects of the diets cannot be directly transposed to human. These studies provide indications of the possible effects of such dietary manipulations on fat and skeletal physiology that may suggest future insights for research of underlying mechanisms.

Larger time-point studies that will include evaluation of the developing bone by using in vivo micro-CT as well as bone strength testing, bone mineral density, histology and histomorphometry and measurement of bone and adipose tissue will 
lead to further insights about post-translational modifications.

\section{Conclusions}

Our results suggest that in the same calorie contents, high fat-low carbohydrate composition in maternal diet during gestation and lactation result a significant changes in bone formation and retroperitoneal fat gene expressions that modulate early-life events to potentiate protection from the risk of bone disorders in female and male offspring adult life. Findings from this study suggest that both the calorie content and macronutrient distribution effect on the next generation. Further research is warranted to explore the exact mechanisms in this process.

\begin{abstract}
Abbreviations
Ctnnb1: Beta-catenin; FASN: Fatty acid synthase; HF-LCD: High fat-low carbohydrate diet; LF-HCD: Low fat-high carbohydrate diet; LPL: Lipoprotein lipase; MSC: Mesenchymal stem cell; OPG: Osteoprotegrin;

PPARx: Peroxisome proliferator-activated receptor gamma; RANK-L: Receptor activator of nuclear factor NF-kB ligand
\end{abstract}

\section{Acknowledgements}

Authors are very thankful from the colleagues in the Breeding Centre of Laboratory Animals at Iran University of Medical Sciences, Tehran, Iran.

\section{Funding}

This research did not receive any specific grant from funding agencies in the public, commercial, or not-for-profit sectors.

\section{Availability of data and material}

Not applicable.

\section{Authors' contributions}

SNM, FSh and FK designed the study. SNM and MBE collected and interpreted data. SNM wrote the first draft of the manuscript. All authors contributed to the discussion and approved the final version of this paper.

\section{Competing interests}

The authors declare that they have no competing interests.

\section{Ethics approval and consent to participate}

The present experimental study was approved by the Animal Research Committee of Tehran University of Medical Sciences, Tehran, Iran.

\section{Author details}

'Department of Cellular and Molecular Nutrition, School of Nutritional Sciences and Dietetics, Tehran University of Medical Sciences, Tehran, Iran. ${ }^{2}$ Department of Nutrition, School of Health, Iran University of Medical Sciences, Tehran, Iran. ${ }^{3}$ Department of Stem Cells and Developmental Biology, Cell Science Research Center, Royan Institute for Stem Cell Biology and Technology, ACECR, Tehran, Iran. ${ }^{4}$ Department of Biochemistry and Nutrition, School of Medicine, Zanjan University of Medical Sciences, Zanjan, Iran.

\section{Received: 27 June 2016 Accepted: 6 October 2016}

Published online: 18 October 2016

\section{References}

1. Oden A, McCloskey E, Kanis J, Harvey N, Johansson H. Burden of high fracture probability worldwide: secular increases 2010-2040. Osteoporos Int. 2015:26:2243-8

2. Roberto CA, Swinburn B, Hawkes C, Huang TT, Costa SA, Ashe M, et al. Patchy progress on obesity prevention: emerging examples, entrenched barriers, and new thinking. Lancet. 2015;385:2400-9.
3. Reid IR. Relationships Between Body Fat and Bone Mass, in: Nutritional Influences on Bone Health, 8th International Symposium. London: Springer; 2013. p. 83-92.

4. Paccou J, Hardouin P, Cotton A, Penel G, Cortet B. The role of bone marrow fat in skeletal health: usefulness and perspectives for clinicians. J Clin Endocrinol Metab. 2015;100:3613-21.

5. Gimble JM, Zvonic S, Floyd ZE, Kassem M, Nuttall ME. Playing with bone and fat. J Cell Biochem. 2006:98:251-66.

6. Teitelbaum SL. Bone resorption by osteoclasts. New York: Science; 2000. p. 1504-8.

7. Horowitz MC, Lorenzo JA. The origins of osteoclasts. Curr Opin Rheumatol. 2004;16:464-8.

8. Duque G. Bone and fat connection in aging bone. Curr Opin Rheumatol. 2008;20:429-34.

9. Karsenty G. Convergence between bone and energy homeostases: leptin regulation of bone mass. Cell Metab. 2006;4:341-8.

10. Sumiyoshi M, Sakanaka M, Kimura Y. Chronic intake of high-fat and highsucrose diets differentially affects glucose intolerance in mice. J Nutr. 2006; 136:582-7.

11. Axen KV, Dikeakos A, Sclafani A. High dietary fat promotes syndrome $X$ in nonobese rats. J Nutr. 2003;133(7):2244-9.

12. Storlien $L H$, Kraegen $E$, Jenkins $A B$, Chisholm DJ. Effects of sucrose vs starch diets on in vivo insulin action, thermogenesis, and obesity in rats. Am J Clin Nutr. 1988;47:420-7.

13. van den Broek M, Leermakers ET, Jaddoe WW, Steegers E, Rivadeneira F, Raat $\mathrm{H}$, et al. Maternal dietary patterns during pregnancy and body composition of the child at age 6 y: the Generation R Study. Am J Clin Nutr. 2015;102: 873-80.

14. MCCurdy CE, Bishop JM, Williams SM, Grayson BE, Smith MS, Friedman JE, et al. Maternal high-fat diet triggers lipotoxicity in the fetal livers of nonhuman primates. J Clin Investig. 2009;119:323-35.

15. Gupta A, Srinivasan M, Thamadilok S, Patel MS. Hypothalamic alterations in fetuses of high fat diet-fed obese female rats. J Endocrinol. 2009;200: 293-300.

16. Gamba CA, Friedman SM, Rodriguez PN, Macri EV, Vacas MI, Lifshitz F. Metabolic status in growing rats fed isocaloric diets with increased carbohydrate-to-fat ratio. Nutrition. 2005;21:249-54.

17. Chen JR, Lazarenko OP, Wu X, Kang J, Blackburn ML, Shankar K, et al. Dietaryinduced serum phenolic acids promote bone growth via p38 MAPK/ $\beta$-catenin canonical Wnt signaling. J Bone Miner Res. 2010;25:2399-411.

18. Rebouças E, Costa JJ, Passos MJ, Passos JR, Hurk R, Silva J. Real time PCR and importance of housekeepings genes for normalization and quantification of mRNA expression in different tissues. Braz Arch Biol Technol. 2013;56:143-54

19. Livak KJ, Schmittgen TD. Analysis of relative gene expression data using real-time quantitative PCR and the 2- $\Delta \Delta C T$ method. Methods. 2001;25:402-8

20. Priego T, Sanchez J, Garcia AP, Palou A, Pico C. Maternal dietary fat affects milk fatty acid profile and impacts on weight gain and thermogenic capacity of suckling rats. Lipids. 2013;48:481-95.

21. Rosa B, Blair H, Vickers M, Dittmer K, Morel P, Knight C, et al. Moderate Exercise during Pregnancy in Wistar Rats Alters Bone and Body Composition of the Adult Offspring in a Sex-Dependent Manner. PLoS One. 2013;8:e82378.

22. Casado-Díaz A, Santiago-Mora R, Dorado G, Gomez J. The omega-6 arachidonic fatty acid, but not the omega-3 fatty acids, inhibit osteoblastogenesis and induces adipogenesis of human mesenchymal stem cells: potential implication in osteoporosis. Osteoporos Int. 2013;24:1647-61.

23. Hofbauer L, Lacey D, Dunstan C, Spelsberg T, Riggs B, Khosla S. Interleukin$1 \beta$ and tumor necrosis factor-a, but not interleukin-6, stimulate osteoprotegerin ligand gene expression in human osteoblastic cells. Bone. 1999;25:255-9.

24. Komori T. Regulation of skeletal development by the Runx family of transcription factors. J Cell Biochem. 2005;95:445-53.

25. Yang K, Cai W, Xu J, Shi W. Maternal high-fat diet programs Wnt genes through histone modification in the liver of neonatal rats. J Mol Endocrinol. 2012:49:107-14.

26. Baron R, Kneissel M. WNT signaling in bone homeostasis and disease: from human mutations to treatments. Nat Med. 2013;19:179-92.

27. Chen X, Garner S, Anderson J. Isoflavones regulate interleukin-6 and osteoprotegerin synthesis during osteoblast cell differentiation via an estrogen-receptor-dependent pathway. Biochem Biophys Res Commun. 2002;295:417-22. 
28. Saika M, Inoue D, Kido S, Matsumoto T. 17ß-Estradiol Stimulates Expression of Osteoprotegerin by a Mouse Stromal Cell Line, ST-2, via Estrogen Receptor-a 1. Endocrinology. 2001;142:2205-12.

29. Chen JR, Lazarenko OP, Wu X, Tong Y, Blackburn ML, Shankar K, et al. Obesity reduces bone density associated with activation of PPARY and suppression of Wnt/ß-catenin in rapidly growing male rats. PLoS One. 2010;5:e13704.

30. Zhao LJ, Jiang H, Papasian CJ, Maulik D, Drees B, Hamilton J, et al. Correlation of obesity and osteoporosis: effect of fat mass on the determination of osteoporosis. J Bone Miner Res. 2008;23:17-29.

31. Pollock N, Laing E, Baile CA, Hamrick MW, Hall DB, Lewis RD. Is adiposity advantageous for bone strength? A peripheral quantitative computed tomography study in late adolescent females. Am J Clin Nutr. 2007:86:1530-8.

32. Cao JJ, Gregoire BR, Gao H. High-fat diet decreases cancellous bone mass but has no effect on cortical bone mass in the tibia in mice. Bone. 2009;44:1097-104.

33. Duivenvoorden I, Teusink B, Rensen PC, et al. Apolipoprotein C3 deficiency results in diet-induced obesity and aggravated insulin resistance in mice. Diabetes. 2005;54:664-71.

34. Berndt J, Kovacs P, Ruschke K, et al. Fatty acid synthase gene expression in human adipose tissue: association with obesity and type 2 diabetes. Diabetologia. 2007:50:1472-80.

35. Idelevich A, Sato K, Baron R. What are the effects of leptin on bone and where are they exerted? J Bone Miner Res. 2013;28:18-21.

36. Takeda S, Elefteriou F, Levasseur R, Liu X, Zhao L, Parker KL, et al. Leptin regulates bone formation via the sympathetic nervous system. Cell. 2002;111:305-17.

37. Elefteriou F, Ahn JD, Takeda S, Starbuck M, Yang X, Liu X, et al. Leptin regulation of bone resorption by the sympathetic nervous system and CART. Nature. 2005;434:14-20

38. Kajimura D, Lee HW, Riley KJ, Arteaga-Solis E, Ferron M, Zhou B, et al. Adiponectin regulates bone mass via opposite central and peripheral mechanisms through FoxO1. Cell Metab. 2013:17:901-15.

\section{Submit your next manuscript to BioMed Central and we will help you at every step:}

- We accept pre-submission inquiries

- Our selector tool helps you to find the most relevant journal

- We provide round the clock customer support

- Convenient online submission

- Thorough peer review

- Inclusion in PubMed and all major indexing services

- Maximum visibility for your research

Submit your manuscript at www.biomedcentral.com/submit 